\title{
EDIFİCATE
}

I Congreso de Escuelas de Edificación y Arquitectura Técnica de España València, 4 y 5 de noviembre de 2021

Escuela Técnica Superior de Ingeniería de Edificación

Universitat Politècnica de València

Doi: https://doi.org/10.4995/EDIFICATE2021.2021.13565

\section{La Exposición de trabajos de clase como motivación para estudiantes de Expresión Gráfica The Exhibition of class works as a motivation for Graphic Expression students.}

\author{
Ruth Pino Suárez y Juan Alejandro Melián Melián \\ Universidad de La Laguna, rpsuarez@ull.edu.es y Universidad de La Laguna, jamelian@ull.edu.es
}

\begin{abstract}
EDIGRÁFICA 2021 has recently been held, a Virtual Exhibition after a long trajectory as a traditional exhibition, with works by students and teachers of Graphic Expression subjects of degrees in the field of building and with international participation.

This is the first time that this exhibition has been carried out in a non-presential way, and this has allowed us to discover new possibilities for the relationship and exchange of learning results.

The experience of this form of exhibition has opened up an innovative way to encourage and promote the work of students in our degrees with a cross-cutting objective: to develop communicative, compositional and expressive skills while carrying out class work associated with the syllabus and/or research in the field of Graphic Expression.

But it also opens up the possibility of interacting with other participants from anywhere, exchanging information, organising on-line presentations within the room and using it as a multi-purpose classroom for teaching.
\end{abstract}

Keywords: Virtual exhibition, class work, research, exchange, interaction.

\section{Resumen}

Recientemente se ha celebrado EDIGRÁFICA 2021, una Exposición Virtual después de una larga trayectoria como exposición tradicional, con trabajos de estudiantes y profesores de asignaturas de Expresión Gráfica de titulaciones del ámbito de la edificación y con participación internacional.

Es la primera vez que se realiza esta exposición de manera no presencial, y esto ha permitido descubrir nuevas posibilidades para la relación y el intercambio de los resultados de aprendizaje.

La experiencia de esta forma de exponer ha abierto una vía innovadora para fomentar y promover la realización de los trabajos del alumnado de nuestras escuelas con un objetivo transversal: desarrollar la capacidad comunicativa, compositiva y expresiva a la vez que realizan trabajos de clase asociados a los temarios y/o a la investigación en materia de Expresión Gráfica. 
La Exposición de trabajos de clase como motivación para estudiantes de Expresión GráficaThe Exhibition of class Works as a motivation for Graphic Expression students.

Pero además abre la posibilidad de interactuar con otros participantes de cualquier lugar, intercambiar información, organizar presentaciones on-line dentro de la sala y usarla como aula multiusos para la docencia.

Palabras clave: Exposición virtual, trabajos de clase, investigación, intercambio, interacción. 


\section{Introducción}

La situación sociosanitaria del último año ha obligado a que tanto las clases como los congresos hayan tenido que adaptarse y encontrar el modo de no paralizarse. Con la exigencia de la no presencialidad, y venciendo múltiples resistencias, hemos descubierto herramientas y vehículos para la comunicación que parecen haber venido para quedarse.

A causa del imperativo de las circunstancias, lo que quizá hubiera tardado décadas en transformarse, se nos ha presentado de golpe, dando un salto sorprendente.

Esto nos ha permitido valorar aquellas herramientas que realmente nos han facilitado ciertos aspectos de la docencia así como las que, en muchos casos por casualidad o necesidad, hemos encontrado para desarrollar otros proyectos académicos y profesionales.

Hace dos años comenzamos a organizar el XV Congreso Internacional de Expresión Gráfica aplicada a la Edificación que, como todas las ediciones desde 1991, iba a ser presencial. Paralelamente al Congreso, siempre se organizaba una exposición denominada EDIGRÁFICA, donde los profesores de Expresión Gráfica de las Escuelas de Arquitectura Técnica e Ingeniería de Edificación presentaban los trabajos de sus estudiantes en paneles de cartón pluma que se colgaban y exponían en algún espacio de la sede del Congreso. En la mayoría de los casos los profesores cargaban con ellos desde sus escuelas de origen, de ahí que a veces sólo llegaran trabajos de ciudades cercanas.

Finalmente hemos celebrado dicho congreso de manera virtual, lo que ha obligado a que la exposición EDIGRÁFICA 2021, en Tenerife, del 27 de mayo al 30 de junio, también fuese virtual.

Del resultado de esta exposición nace esta propuesta docente gracias a la múltiples posibilidades que la aplicación nos abre hacia la interacción y el encuentro de estudiantes, profesores y participantes de otras nacionalidades.

\section{Objetivos}

A la vista del resultado de la Exposición Virtual que ha sido montada para EDIGRÁFICA 2021, analizando sus fortalezas y debilidades, se pretende proponer esta iniciativa participativa como un reto motivacional para profesores y estudiantes de estas materias.

La visita a la Exposición permite conocer los trabajos de otros estudiantes e investigadores, erigiéndose en sí misma como una herramienta de aprendizaje que pone en valor a la Expresión Gráfica y muestra una realidad profesional para futuros graduados.

Sin embargo, esta propuesta trata de promover la acción en cuanto a la participación en las futuras exposiciones, conociendo este objetivo en el momento de realizar los ejercicios prácticos de clase, añadiendo el reto expositivo al conjunto de competencias e integrando un aprendizaje basado en proyectos que sirva para impulsar la motivación de los estudiantes. 


\section{Desarrollo de la innovación. EDIGRÁFICA 2021}

Con motivo de la celebración del XV Congreso Internacional de Expresión Gráfica aplicada a la Edificación: Redibujando el Futuro - APEGA 2021, en Tenerife, se inauguró el pasado 27 de mayo la Exposición EDIGRÁFICA 2021.

Siendo la primera vez que se hace virtual y a la vista de las ventajas que han sobresalido tras esta experiencia, la Asociación de Profesores de Expresión Gráfica Aplicada a la Edificación tiene la convicción de que esta forma de exponer los trabajos de EDIGRÁFICA va a ser determinante en los Congresos futuros de APEGA.

\subsection{Antecedentes}

Los Congresos de APEGA comenzaron a organizarse en el año 1991 en las que se denominaron Primeras Jornadas de Expresión Gráfica Aplicada a la Edificación, en Sevilla, y desde entonces se vienen celebrando cada dos años ininterrumpidamente con carácter nacional hasta 2010 en que pasó a ser internacional y alcazó un hito de participación.

APEGA, como asociación, nació con el objetivo principal de fomentar la solidaridad y defensa de sus miembros, teniendo por objeto cuantas actividades tiendan a impulsar, potenciar, orientar, coordinar, desarrollar y promover en sus vertientes científicas y técnicas, la enseñanza y la divulgación de la Expresión Gráfica Arquitectónica en las Escuelas Técnicas Superiores de Ingeniería de Edificación, de Edificación, Politécnicas o con cualquier otra denominación, en las que se imparta la enseñanza de esta área de conocimiento y que impartan la titulación de grado que habilita a la profesión de Arquitecto Técnico (APEGA)

A partir de 1995, en el Congreso celebrado en La Coruña, se instauró EDIGRÁFICA, con el fin de exponer los trabajos de estudiantes y profesores de las diferentes escuelas de España, lo que contribuyó a la visibilidad de los resultados de docencia y aprendizaje en las diversas materias de la Expresión Gráfica.

La exposición EDIGRÁFICA pretende seguir conservando su espíritu inicial de constituir una muestra de logros conseguidos por el profesorado y alumnado vinculado a la Expresión Gráfica Arquitectónica e Ingeniería de la Edificación, perdurando en el tiempo y reafirmándose como instrumento de difusión nacional e internacional en todas sus dimensiones.

La historia de las quince ediciones de EDIGRÁFICA puede demostrar cómo la Expresión Gráfica aplicada a la Edificación ha ido evolucionando y adaptándose a los cambios que las nuevas tecnologías han ido imponiendo.

\subsection{Contexto Profesional}

Tanto los Congresos como las Exposiciones EDIGRÁFICA se enmarcan en el contexto de la docencia de Profesores de las áreas de Expresión Gráfica Arquitectónica que se constituyeron como Asociación en 1993, cuando la carrera y las asignaturas tenían el mismo nombre y mismo plan de estudios en casi todas la Universidades de España. 
La muestra de los trabajos de los estudiantes dirigidos por el profesorado de nuestras escuelas a través de EDIGRÁFICA ha permitido dar testimonio, y pretende seguir dándolo, de cómo se representa el mundo en construcción, el construido y el desaparecido, desde antes de la incorporación de los primeros dibujos asistidos por ordenador hasta las más novedosas metodologías actuales.

EDIGRÁFICA cuenta de forma visual la historia de la Expresión Gráfica aplicada a la Edificación y, por tanto, narra parte de la historia del mundo.

Profesionalmente los trabajos expuestos demuestran cómo los estudiantes aplican los conocimientos y los avances tecnológicos de aplicación en la topografía, levantamiento de planos, escaneados, modelado y recogida de datos, gestión de la información gráfica y de proyectos que tanta importancia tiene en el desarrollo posterior de nuestros egresados.

\subsection{Premios a los mejores trabajos}

Más tarde se establecieron los Premios a los mejores trabajos de EDIGRÁFICA que motivan y reconocen los méritos de los participantes en la Exposición.

En honor al Profesor D. Juan Manuel Raya Urbano, fallecido en 2008, estos premios comenzaron a llevar su nombre a partir del congreso de Alicante, en 2010.

Fue Catedrático de Dibujo Arquitectónico II de la Escuela Universitaria de Arquitectura Técnica de Sevilla y gran promotor e impulsor de la Asociación APEGA, así como de los congresos y actividades que ésta realiza.

Para EDIGRÁFICA 2021, observando que la realización virtual permitiría incluir en la exposición a un mayor número de participantes extranjeros, se abrió la posibilidad de abarcar trabajos docentes y de investigación que, sin salirse de la línea temática, tuvieran la visión y el desarrollo metodológico de otras latitudes. Los participantes en EDIGRÁFICA han presentado los trabajos con la debida antelación, siguiendo estrictamente instrucciones con el fin de garantizar la calidad de las imágenes y el anonimato de los autores para su valoración por parte del Jurado de los Premios Juan Manuel Raya.

El rigor de los trabajos ha permitico la realización de una Exposición y un Catálogo con excelente calidad de imagen tanto en la versión digitales como impresa. Del mismo modo, los premiados han podido figurar como tal en todos los documentos gráficos que se han publicado desde el mismo momento de la inauguración de EDIGRÁFICA 2021.

Estos premios y la visibilidad de los trabajos de todos lo participantes que les otorga su presencia en la web, así como su inclusión en el correspondiente Catálogo, son un aliciente y un mérito que pueden motivar a los futuros estudiantes de Expresión Gráfica y sus profesores a concursar en las próximas ediciones.

\subsection{Catálogo de la Exposición}

Es una publicación que recoge la totalidad de los trabajos que participan en EDIGRÁFICA, y donde además se destacan a los premiados por el jurado de los Premios Juan Manuel Raya, 
lo que supone un considerable mérito para los autores, en su mayoría grupos de estudiantes con sus respectivos profesores o tutores de trabajos de investigación.

Se ha iniciado una colección de Catálogos de EDIGRÁFICA gracias al impulso y la iniciativa de los comisarios que organizaron la Exposición para el XIV Congreso de APEGA que se celebró en Sevilla en 2019, siendo el primero de la colección y que lleva por título Catálogo de la Exposición EDIGRÁFICA 2019 (Rincón Millán y Díaz Cañete, 2020).

El Catálogo de la Exposición EDIGRÁFICA 2021, realizado en Tenerife, suma el segundo volumen de la incipiente colección (Pino Suárez y Melián Melián, 2021). Este catálogo está disponible en formato digital en el Repositorio Institucional de la Universidad de La Laguna (https://riull.ull.es/xmlui/handle/915/23955).

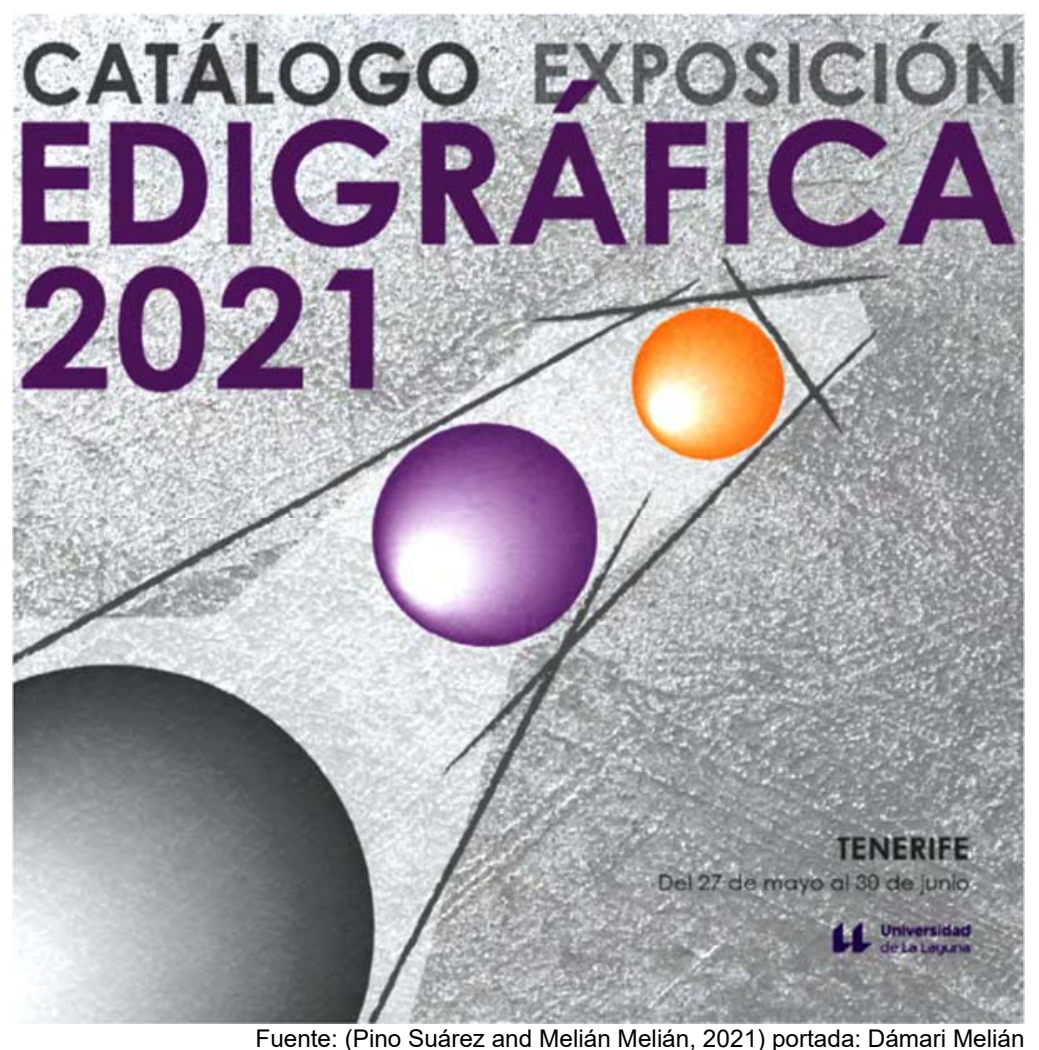

Fig. 1 Portada Catálogo Exposición EDIGRÁFICA 2021. 


\subsection{Exposición Virtual}

El 27 de mayo de 2021 se inauguró la Exposición EDIGRÁFICA 2021 de Tenerife, coincidiendo con el inicio del Congreso.

En la página web del congreso se preparó una exposición multimedia, mediante la cual se podían ver todos los trabajos pinchado alternativamente en cada título y accediendo a la información enlazada así como a cada uno de los paneles para ser visualizados en detalle con total calidad de imagen. Para esta muestra de EDIGRÁFICA se empleó la aplicación Genial.ly.

En la pantalla de inicio se encuentran los títulos de los trabajos presentados, siguiendo una numeración que, posteriormente se hizo coincidir tanto en la Exposición Virtual como en la edición del Catálogo.

Se puede observar la distinción entre las dos modalidades en que está dividida EDIGRÁFICA, docencia e investigación, de modo que los Premios Juan Manuel Raya otorgan un único galardón por modalidad y se reserva la opción de conceder dos accésits.

\section{EXPOSICIÓN EDIGRÁFICA 2021}

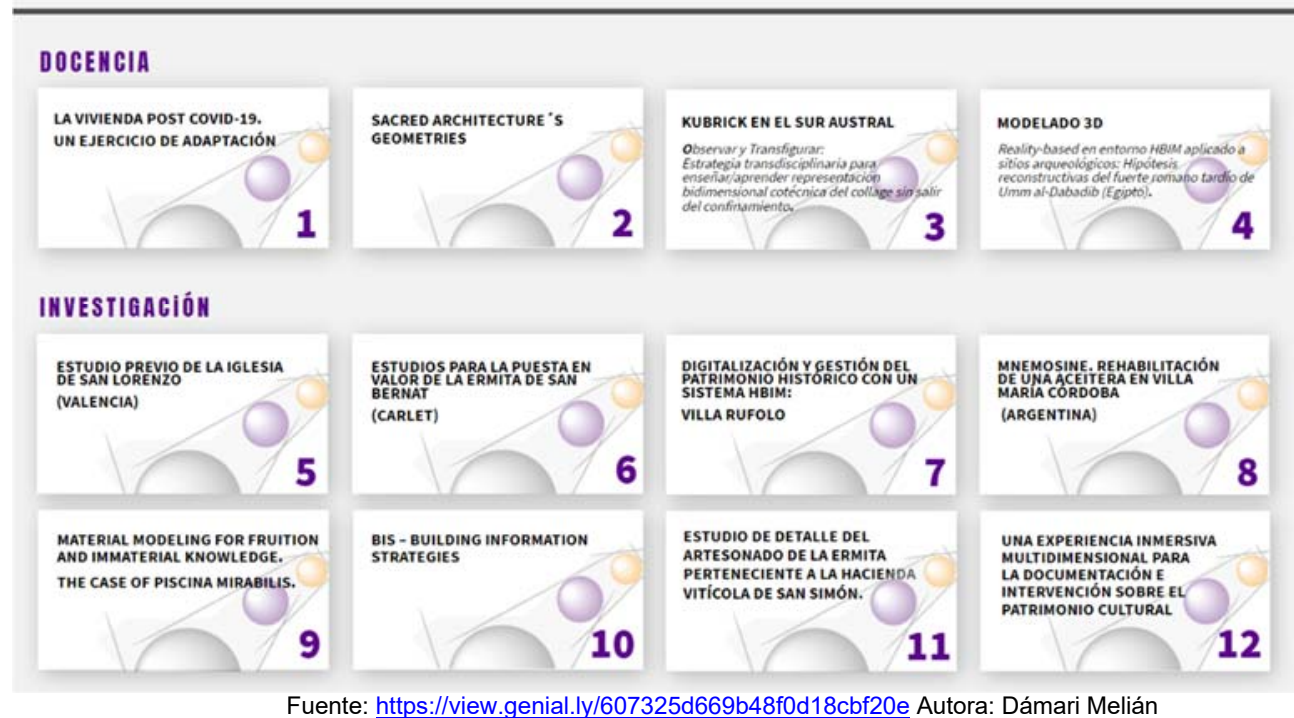

Fig. 2 Página de Inicio de EDIGRÁFICA donde figuran todos los trabajos presentados al concurso y expuestos en la sala virtual.

Pero la Exposición más dinámica y más atractiva ha sido la que finalmente ha permitido tener la posibilidad de pasear virtualmente por la Sala de Exposiciones donde se han colocado todos los paneles, enviados en formato jpeg y con resolución 600 ppp. 
Presentada y accesible desde la página web del Congreso, todo el público al que se le haya hecho llegar el enlace, puede visitarlo desde su ordenador y moverse con los mandos indicados, del mismo modo que un videojuego. Se incluye la posibilidad de que cada visitante pueda personalizar su propio "avatar", poner su nombre y encontrarse por la sala con otros avatares e interactuar con ellos como si se tratara de una visita real.

\subsubsection{Soporte técnico}

Para montar la exposición se ha utilizado la plataforma Mozilla Hubs (Hubs - Private, virtual 3D spaces in your browser). Es una plataforma gratuita que permite diseñar los entornos de forma bastante intuitiva, con la herramienta Spoke (Spoke by Mozilla) similar a muchos programas que se usan en Expresión Gráfica.

No obstante, usar Hubs de manera gratuita tiene sus limitaciones, ya que no se pueden personalizar numerosos aspectos de las salas, como por ejemplo la capacidad. Para la Exposición EDIGRÁFICA 2021 se han implementado algunas opciones para la personalización y se ha montado en una instancia de Amazon Web Services (AWS Cloud Computing - Servicios de informática en la nube), lo que ha requerido la ayuda de técnicos con conocimientos más avanzados. Además se ha sido necesario asumir los costes de todos los servicios asociados para que la instancia funcione lo que dure la exposición.

\section{EXPOSICIÓN EDIGRÁFICA 2021}

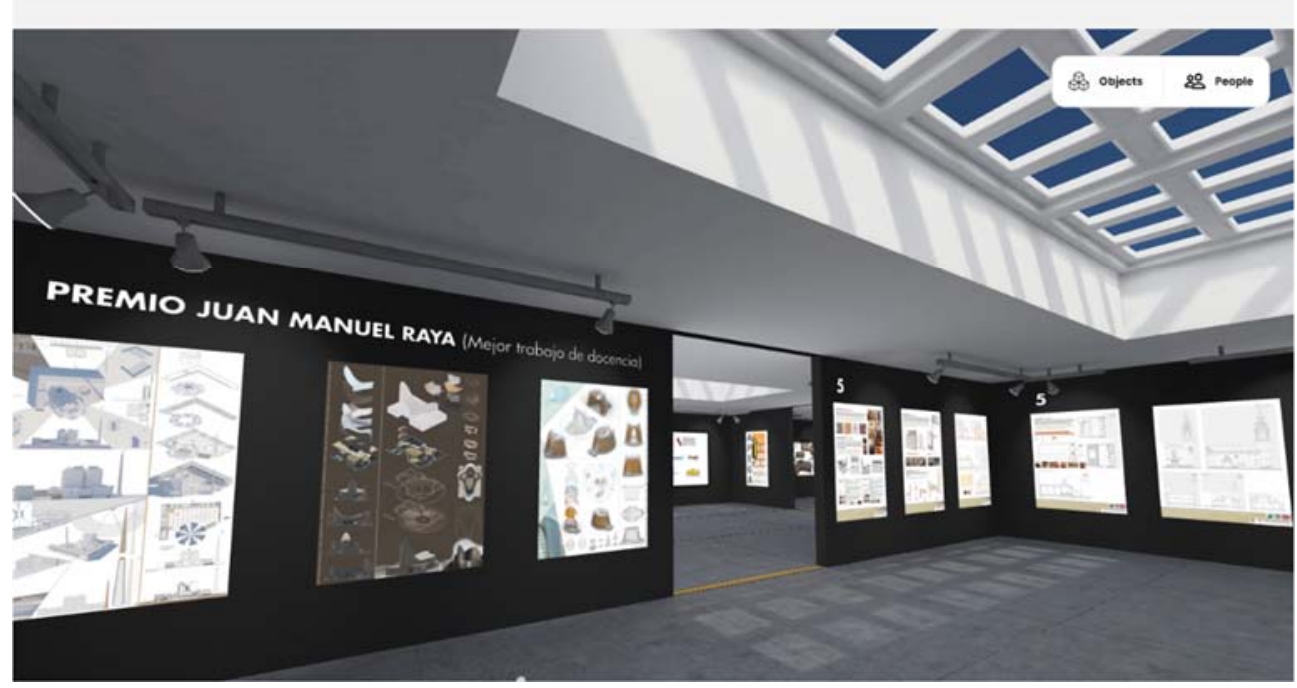




\section{EXPOSICIÓN EDIGRÁFICA 2021}

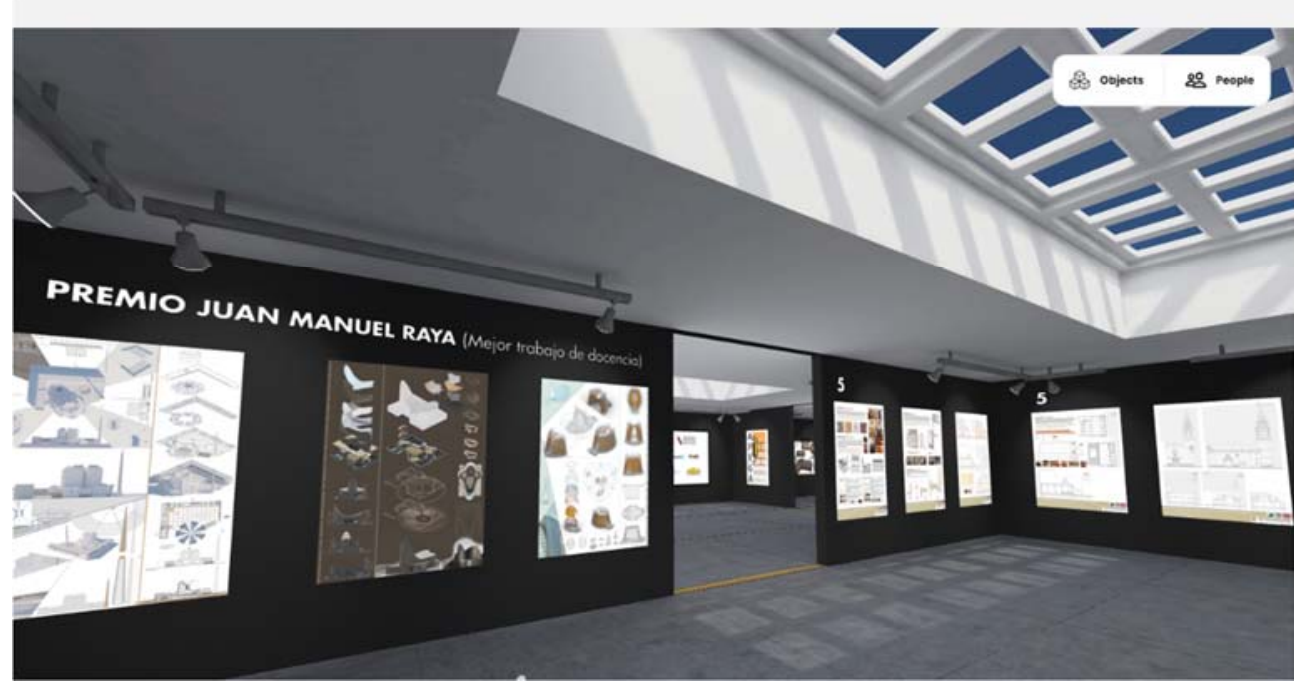

Fuente: elaboración propia

Fig. 3 Capturas de pantalla de la Exposición Virtual EDIGRÁFICA 2021-Tenerife, interactiva, accesible del 27 de mayo al 30 de junio de 2021

\subsubsection{Fortalezas}

A todos los efectos, esta Exposición Virtual, ha cumplido con la expectativas de una exposición al uso tradicional, teniendo en cuenta que los principales usuarios y visitantes iban a ser los propios participantes y los asistentes al Congreso.

Pero desde que se hiciera el llamamiento a la participación en EDIGRÁFICA 2021 a los diferentes colectivos de profesores y universidades incluyendo otros países europeos y latinoamericanos, se tomó conciencia de que al ser virtual sería muy fácil la participación internacional. Pero también la nacional, puesto que no se hacía necesario el envío de paneles en cartón pluma para su montaje y desmontje, ni tampoco prever lugares en Tenerife donde hacer las impresiones, etc.

Enumeramos a continuación todas las cualidades que han aflorado como fortalezas en esta modalidad expositiva:

- Hay cabida para un mayor número de trabajos sin necesidad de limitarlo a un espacio concreto.

- $\quad$ No hay que montar los paneles físicamente y desmontarla al finalizar.

- Se facilita la participación en la Exposición, sin costes de envío ni de impresión. Además los participantes no están obligados a asistir al Congreso. 
- Se promueve el intercambio de información y conocimiento entre los profesores y los estudiantes de diferentes países.

- La exposición resulta divertida de visitar y se asemeja a un juego, donde los participantes pueden interactuar unos con otros y dirigirse hacia un panel $u$ otro $y$ comentarlo.

- $\quad$ Los spónsors se pueden hacer más visibles y tener enlace a sus páginas web.

- El alcance y visibilidad de la Exposición a trevés de internet la convierte en potencialmente global.

- Se puede acceder a la sala en cualquier momento del día, noche o día de la semana y desde cualquier parte del mundo.

- Profesores y estudiantes de cualquier nacionalidad pueden citarse en la sala y realizar la visita en grupo guiados por el profesor. Se puede invitar a los autores de los trabajos a dar charlas dentro de la misma exposición.

- Los méritos de los participantes quedan igualmente acreditados con los certificados correspondientes y la publicación del Catálogo de la Exposición.

\subsubsection{Debilidades}

Es posible que las debilidades de este tipo de exposición sean las mismas que subyacen en todos los eventos que nos hemos ido viendo obligados a realizar on-line, como lo ha sido el propio Congreso de APEGA 2021.

Lo más destacable es la falta del contacto social y las relaciones humanas. No hay duda de que existe una frialdad y distancia entre las personas que se encuentran en cualquier reunión virtual. Pero más aun cuando la esencia de los congresos como los que organiza APEGA radica en conocer personalmente a los ponentes y prolongar los coloquios y discusiones de manera informal durante los momentos de ocio y descanso. Eso es insustituible.

Sin embargo, en el caso de EDIGRÁFICA como espacio expositivo, realmente en la modalidad presencial no representa un lugar de encuentro, sino más bien de paso o de paseo prácticamente individual, más que colectivo. Esta razón es una debilidad de la exposición tradicional, lo que se traduce en una fortaleza más de la exposición virtual.

No obstante, podemos decir que existen algunos puntos débiles:

- El montaje de la exposición virtual podría tener un coste muy bajo, sin embargo requiere destreza y soltura con las plataformas necesarias.

- Si se necesita ampliar capacidad o implementar servicios específicos así como un lugar donde alojar la exposición, hay que prever y asumir ciertos gastos y el apoyo de técnicos más experimentados.

- Puede resultar difícil acceder a visitantes que no estén familiarizados con el uso de estas plataformas o de internet.

- La exposición no está físicamente instalada en la Sede del Congreso, habitualmente es en el vestíbujo de la Escuela de Arquitectura Técnica a la que haya correspondido la organización, y el alumnado no se la encuentra cuando va a su clase mientras se 
celebra el un congreso de Expresión Gráfica en su centro, lo que se traduce en una alta visibilidad global en las redes, pero nula visibilidad local.

- La Exposición no se nutre tanto de los trabajos de profesores y estudiantes que pertenecen a la Universidad que acoge el congreso $y$, por extensión, a EDIGRÁFICA.

- Al abrir EDIGRÁFICA a la internacionalización también se produce un salto diferencial en el tipo de trabajos que se presentan desde otras universidades extranjeras, cuyos estudiantes son de doctorado o de carreras afines a la Arquitectura Técnica pero que han focalizado su aprendizaje en campos muy concretos y con disponibilidad de medios tecnológicos que los hacen diferenciarse.

- Esto puede resultar desmotivador y en ese sentido se apunta como una debilidad. Sin embargo, visto el resultado de esta primera exposición virtual, parece que también debe ser un estímulo para profundizar en otros modos de hacer e impulsar la excelencia de los trabajos de Expresión Gráfica de los futuros profesionales de la Edificación.

\section{Resultados. Propuesta Docente}

Por todo lo explicado, hemos considerado que un espacio expositivo virtual como EDIGRÁFICA 2021, se presenta como una oportunidad para desarrollar experiencias docentes con los estudiantes de Expresión Gráfica que se forman actualmente en nuestras escuelas.

Aprovechando que EDIGRÁFICA se celebra cada dos años, es posible organizar a los estudiantes desde segundo curso hasta el último, donde se preparan los Trabajos Fin de Grado, retándoles a que presenten sus trabajos en esta Exposición Virtual Internacional.

Las razones por las que esta propuesta podría ser de gran atractivo para los estudiantes se basan en objetivos de corto, medio y largo plazo, lo que requerirá poner en práctica diferentes fases de planificación y planteamientos del trabajo.

Pero lo que se busca realmente es que los estudiantes, dirigidos por el profesorado, asuman el reto y descubran una motivación más allá del resultado académico.

\subsection{La Motivación}

Una cuestion interesante a tener en cuenta por el profesorado, derivada de las recomendaciones tomadas de la investigación de Stipek (1988) para lograr la motivación de los alumnos al aprendizaje, hace referencia a que el profesor debe propiciar un contacto de los alumnos con el mundo real exponiendo casos reales de su ámbito profesional.

Los trabajos que se exponen en EDIGRÁFICA, especialmente los de investigación, son fieles reflejos de lo que se hace actualmente para realizar levantamientos de planos, topografías, análisis de patologías, comparaciones históricas del patrimonio, modelados con supuestos 
pasados y futuros, sistemas de información geográficos, combinaciones entre unos conocimientos y otros, etc.

Aunque sólo fuera para conocer en qué consiste la Exposición EDIGRÁFICA, los alumnos de cualquier curso que la visiten, estarían aprendiendo las cosas que se pueden hacer con la formación en Expresión Gráfica que reciben y con la que aún desconocen.

El profesor debe ser el primer implicado en el reto, y considerar qué motivación presenta su alumnado con el fin de estimular no sólo el logro de la recompensa externa como el aprobado del curso sino también la valoración social y el aumento de la capacidad y profundidad del conocimiento (Parrales Rodríguez y Solórzano Salas, 2014).

De modo que esta experiencia docente, que aún no se ha puesto en práctica, tiene que contar con la disposición de un profesor o grupo de profesores que mantenga el reto vivo entre su alumnado motivado.

Pese a que un elevado porcentaje de alumnos da más importancia al hecho de obtener los resultados académicos apetecidos que al hecho de aprender, existe la motivación intrínseca de los estudiantes, la que les nace de forma natural, que consigue mejor rendimiento y aprendizaje para el futuro. $\mathrm{Y}$, aunque requiere más esfuerzo y dedicación por parte de los estudiantes, repercute en una mejora del proceso de enseñanza-aprendizaje y sienta las bases para un aprendizaje significativo, lo que enlaza directamente con los principios educativos del EEES (Crespo Fernández y Martínez Lirola, 2008), además de aportar importantes ventajas en cuanto al rendimiento y la salud psíquica del estudiante (Mas Tous y Medinas Amorós, 2007).

El desarrollo por parte de los docentes de estrategias de motivación en sus actividades, generando simples desafíos vinculados con problemas reales que generen interés en los alumnos por llegar a una resolución satisfactoria y por aprender, redundará en el logro de un mayor nivel de compromiso por parte de dichos alumnos en la solución de los problemas planteados y en la construcción de sus propios conocimientos (Hector Ardisana, 2012).

Teniendo en cuenta las consideraciones de numerosos expertos en motivación, se puede entender que los estudiantes estén abiertos a preparar sus trabajos de clase para ser expuestos en una Exposición Virtual como EDIGRÁFICA con el fin de:

- Participar en una Exposición que convoca a otros estudiantes de carreras similares a nivel nacional e internacional.

- Optar a alguno de los Premios Juan Manuel Raya.

- Publicar sus trabajos en un Catálogo.

Para el profesorado, esta propuesta puede ser interesante a la hora de plantear trabajos en grupo y promover un aprendizaje basado en el Proyecto Expositiva, lo que obligaría a que los estudiantes desarrollaran sus competencias transversales en la línea de liderazgo, resolución de problemas, trabajo colaborativo, etc. 
Tendrán que seguir unas normas y plazos de presentación de los trabajos, organizar los paneles en los formatos exigidos con la información o los dibujos que quieran incluir, establecer el relato gráfico de su idea a transmitir, temática o secuencia de aprendizaje.

Finalizado el Proyecto tendrán la recompensa de ver el resultado de su trabajo en una publicación y una Exposición donde encontrarse e incluso divertirse como con un videojuego, a la vez que ellos mismos serán promotores y divulgadores tanto de su aprendizaje como del sentido de la Expresión Gráfica en la sociedad.

\subsection{El espacio expositivo: interactivo-docente-internacional}

Una vez que la Exposición está montada, se abre una nueva faceta que resulta de la versatilidad de la Sala Virtual de la Exposición.

- Es un espacio interactivo, abierto y global, ya que tiene libre acceso y funciona como una sala presencial excepto porque las personas que vemos son unos muñecos con un número asociado. Son visitantes que están viendo la exposición al mismo tiempo y que, si lo desean, pueden hablar mediante el micrófono que se activa al acceder a la sala.

- Es un espacio docente donde hacer reuniones o dar clases on-line, lo que permite salir de la rutina y mostrar a los demás estudiantes cómo se trabaja en otras universidades, fomentando el interés y la curiosidad por el conocimiento de las herramientas que se utilizan en Expresión Gráfica para lograr distintos objetivos en la representación arquitectónica.

- Es un espacio internacional, o pretende serlo en las sucesivas ediciones, ya que participan estudiantes y profesores del extranjero, especialmente de Italia, y alguno de Chile y Argentina. Este hecho, que confiere a EDIGRÁFICA un nuevo rasgo de internacionalización y apertura, supone una gran oportunidad para fomentar relaciones y contactos fuera de nuestras fronteras.

\section{Conclusión}

La experiencia llevada a cabo en EDIGRÁFICA 2021 como exposición virtual frente a la presencial, ha abierto una via innovadora que debe servir para fomentar y promover la realización de trabajos de los estudiantes de nuestras escuelas con un objetivo transversal: desarrollar la capacidad comunicativa, compositiva y expresiva a la vez que se les motiva para realizar trabajos de clase asociados a los temarios y/o a la investigación en materia de Expresión Gráfica.

Una buena oportunidad para poner en marcha esta propuesta didáctica entre los profesores y estudiantes de Expresión Gráfica de nuestras escuelas, sería la próxima Exposición EDIGRÁFICA 2023, aprovechando que tiene los medios y la infraestructura para que los trabajos de clase sean expuestos de una forma sostenible y global; más aún cuando las valoraciones de los usuarios de esta primera exposición virtual han sido altamente positivas. 
Esta experiencia docente se podrá completar con la organización interuniversitaria de encuentros en la Sala de Exposiciones, estableciendo un programa de eventos que también promuevan reuniones con estudiantes y profesores de otros países.

\section{Referencias}

APEGA Asociación de Profesores de Expresión Gráfica Aplicada a la Edificación, Objetivos. $<$ https://apega.blogs.upv.es/objetivos/> [Consulta: 28 de Junio de 2021]

AWS | CLOUD COMPUTING - Servicios de informática en la nube. <https://aws.amazon.com/es/> [Consulta: 28 de Junio de 2021]

CRESPO FERNÁNDEZ, E. y MARTÍNEZ LIROLA, M. (2008) "Convergencia Europea, motivación y actuación docente en el aula universitaria", en Revista de Enseñanza Universitaria, (31), pp. 416.

HECTOR ARDISANA, E. F. (2012) "La motivación como sustento indispensable del aprendizaje en los estudiantes universitarios.", en Pedagogía Universitaria, XVII(4), pp. 13-27.

HUBS. Private, virtual 3D spaces in your browser. <https://hubs.mozilla.com/> [Consulta: 28 de Junio de 2021]

MAS TOUS, C. y MEDINAS AMORÓS, M. (2007) "Motivaciones para el estudio en universitarios", en Anales de Psicología. Servicio de Publicaciones de la Universidad de Murcia, 23(1), pp. 17-24.

PARRALES RODRÍGUEZ, S. y SOLÓRZANO SALAS, J. (2014) "Motivación y estrategias de aprendizaje del estudiantado de la Escuela de Orientación y Educación Especial", en Revista electronica: Actualidades investigativas en educacion. Universidad de Costa Rica, 14(1), pp. 1-20.

PINO SUÁREZ, R. y MELIÁN MELIÁN, J. A. (2021) Catálogo Exposición EDIGRÁFICA 2021. Universidad de La Laguna. <http://riull.ull.es/xmlui/handle/915/23959> [Consulta: 29 de Junio de 2021]

RINCÓN MILLÁN, M. D. y DÍAZ CAÑETE, P. (2020) Catálogo Exposición EDIGRÁFICA 2019. Editorial Círculo Rojo. Sevilla, España.

SPOKE by Mozilla. <https://hubs.mozilla.com/spoke> [Consulta: 28 de Junio de 2021]

STIPEK, D. (1988) Motivation to learn: From theory to practice. E. Cliffs. New York, USA: Prentice Hall. 